\title{
Deepened extinction following compound stimulus presentation: Noradrenergic modulation
}

\author{
Patricia H. Janak ${ }^{1,2}$ and Laura H. Corbit ${ }^{1,3,4}$ \\ ${ }^{1}$ Ernest Gallo Clinic and Research Center, University of California at San Francisco, Emeryville, California 94608, USA \\ ${ }^{2}$ Department of Neurology, University of California at San Francisco, San Francisco, California 94158, USA; ${ }^{3}$ School of Psychology, \\ The University of Sydney, Sydney, New South Wales, 2006 Australia
}

\begin{abstract}
Behavioral extinction is an active form of new learning involving the prediction of nonreward where reward has previously been present. The expression of extinction learning can be disrupted by the presentation of reward itself or reward-predictive stimuli (reinstatement) as well as the passage of time (spontaneous recovery) or contextual changes (renewal). The following experiments replicated the demonstration that presenting multiple previously rewarded stimuli in compound during extinction enhances extinction learning. To explore the pharmacological basis for this we next examined the effects of pharmacological treatments that either facilitated or blocked noradrenergic activity to test the hypothesis that increased noradrenergic activity at the time of extinction training would improve, whereas blockade of noradrenergic activity would impair the extinction of appetitive stimulus-reward memories. Different groups of rats were trained in a discriminative stimulus paradigm to lever-press for food reward. Once stable responding was achieved, responding was extinguished for $2 \mathrm{~d}$. Prior to a third extinction session, rats received systemic administration of either saline, yohimbine ( $\alpha 2$ antagonist), atomoxetine (norepinephrine reuptake inhibitor), or propranolol ( $\beta$-receptor antagonist). Spontaneous recovery of responding to the stimuli was tested $4 \mathrm{wk}$ later. Our results indicate that increasing noradrenergic activity during extinction augments extinction learning resulting in less recovery of responding at test. These results have important implications for models of relapse to drug seeking and the development of extinction-based therapies.
\end{abstract}

Current theories of associative learning suggest that extinction is not the forgetting or "unlearning" of the original association, but rather involves active new learning involving the prediction of nonreward (Pavlov 1927; Delamater 1996; Rescorla 1996, 1997). Extinction learning is notoriously labile and easily disrupted. Even following extinction training, which may far exceed the duration of the original excitatory conditioning, the expression of extinction is easily disrupted by the passage of time (spontaneous recovery) (Rescorla 1997), stress or arousal (disinhibition) (Pavlov 1927; Horns and Heron 1940), the presentation of the previously trained reinforcer or stimuli associated with that reinforcer (reinstatement) (Rescorla and Heth 1975), or changes in the test context (renewal) (Bouton and King 1983). The aim of the current series of experiments was to examine approaches that might enhance extinction learning.

The impact of a reinforcing event, such as reward delivery, depends on the degree to which that reinforcer is well signaled; a reinforcer that is preceded by a well-conditioned excitatory stimulus has a reduced ability to produce new learning (Kamin 1968). Observations such as this have lead to the development of errorcorrection models of learning (e.g., Rescorla and Wagner 1972), which postulate that learning will occur when there is a discrepancy between what is expected based on the presence of predictors (i.e., conditioned stimuli; CS) and the reinforcer that actually occurs (reward, in appetitive conditioning). These models make predictions not only about what will happen with reinforcement, but also about what learning will occur in the case of nonreinforcement, or extinction. Within this framework, the concurrent presence of an excitatory stimulus at a time when a

\footnotetext{
${ }^{4}$ Corresponding author.
}

E-mail laura.corbit@sydney.edu.au; fax +61290365223.

Article is online at http://www.learnmem.org/cgi/doi/10.1101//m.1923211. response is nonreinforced would result in a particularly large prediction error, and hence should enhance the extinction of that response when it is not reinforced. In line with that prediction, Rescorla (2000) demonstrated that extinguishing a previously rewarded response in the presence of an additional excitatory stimulus resulted in significantly less subsequent responding at test than extinguishing the response either alone or in the presence of a neutral stimulus. Reberg (1972) made the interesting observation that even when two stimuli have themselves been extinguished individually, presenting them together, in compound, yielded greater responding than was observed to either stimulus alone, suggesting again that extinction does not entirely eliminate the original excitatory conditioning, and compound presentation potentially reintroduces a prediction error, which could in turn lead to further learning. This possibility was explored by Rescorla (2006) where he reported that nonreinforcement of a compound of stimuli augmented extinction when the components were later tested compared with a stimulus that was presented alone for the same number of extinction trials, suggesting that nonreinforcement of a compound stimulus further engaged learning mechanisms at the time of nonreinforcement, and thus, deepened the resultant extinction learning.

While error correction models focus on the conditions under which learning will occur, they do not necessarily specify the mechanism. When an animal is confronted with a discrepancy between what is expected and what occurs, in addition to generating a prediction error, the experience itself may be arousing and engage the attentional and neural mechanisms responsible for establishing long-term memories.

Emotional memories are often better remembered than neutral ones (e.g., Dolan 2002), and neutral stimuli may be better remembered when encoded in an emotional context (Sterpenich et al. 2006). A hallmark of many emotional experiences is the 
release of neural and hormonal modulators that produce characteristic arousing effects. Many of these neural and hormonal modulators, including norepinephrine (NE), have been proposed to modulate learning and memory formation (McGaugh 2004; Arnsten 2009). For example, pharmacological treatments that increase the NE response to stimuli facilitate episodic memory formation (O'Carroll et al. 1999; Southwick et al. 2002). In contrast, treatments that block postsynaptic NE effects decrease the memory benefit typically seen with emotional stimuli (Cahill et al. 1994; Berlau and McGaugh 2006). Importantly, arousal and associated noradrenergic effects may be particularly important for modifying performance when reward contingencies or reward value change as is the case in extinction (e.g., Sara et al. 1994; Usher et al. 1999).

The aim of the current series of experiments was to examine ways that extinction learning might be enhanced through manipulations of stimulus presentation and by pharmacological modulation of the noradrenergic system. Rats were trained in a discriminative stimulus paradigm wherein performance of a leverpress response in the presence of one of three discrete stimuli resulted in the delivery of food reward. Thereafter, rats were extinguished and the efficacy of the extinction training was later assessed by examining spontaneous recovery of responding. We show that extinction of a stimulus compound improves extinction of the elements of that compound relative to extinction of a single stimulus. Further, we attempted to either mimic or block the benefit to extinction found with compound stimulus presentation by administering drugs that modulate noradrenergic signaling. To the extent that the benefit observed with compound stimulus presentation involves noradrenergic signaling, we predicted that drugs that increase NE should improve, whereas drugs that block NE should impair extinction revealed as changes in the degree of spontaneous recovery of responding at test. Consistent with these predictions we demonstrate that administration of the $\alpha 2$-antagonist yohimbine or the norepinephrine transporter blocker atomoxtetine augmented the extinction of a single stimulus. In contrast, administration of the $\beta$-adrenergic antagonist propranolol diminishes the benefit of compoundstimulus extinction. These findings suggest that extinction of reward-paired stimuli can be modulated by manipulations of noradrenergic signaling.

\section{Results}

\section{Experiment 1: Compound stimulus presentation}

The aim of this experiment was to replicate Rescorla's (2006) finding that presentation of a compound of extinguished stimuli deepens extinction of the elements of that compound compared with extinction of a stimulus alone. Rats received training in which three separate stimuli (white noise, clicker, and light) signaled the availability of a food pellet following an instrumental lever-press response ( $7 \mathrm{~d}, 24$ trials per day). Thereafter, all of the stimuli underwent the same amount of extinction training; the critical difference was that following initial extinction of each stimulus alone (two sessions), two of the stimuli were presented together (compound) during further extinction trials, whereas the remaining stimulus continued to be presented alone. Of note, by previously extinguishing the individual stimuli, even if additional nonreinforcement of the individual is expected to be without further effect, the reintroduction of a prediction error with the stimulus compound permits further learning and thus allows the possibility of achieving extinction beyond what could be produced by presentations of the stimuli alone. Further, the initial extinction reduces the chances that a more salient stimulus may overshadow a less salient one and thus retard rather than enhance extinction (for a detailed discussion, see Rescorla 2006). Spontaneous recovery of responding was assessed 1 and 4 wk after the final extinction session. Table 1 summarizes the experimental design. On the final day of acquisition the mean rates $( \pm$ SEM) of lever pressing were 4.0 (0.6), 12.9 (1.1), 16.4 (1.2), and $15.6(1.3)$ responses per trial for the pre-CS, light, noise, and clicker, respectively. There were no reliable betweenstimulus differences in response rates.

Over the course of extinction responding decreased for all three stimuli. Prior to the differential treatment of the noise and clicker, responding during these stimuli was similar and low for the to-be compound and to-be single stimulus (Fig. 1A). There were no reliable between-stimulus differences in response rates $(F<1)$. On the third extinction day, one of the auditory stimuli was presented along with the light stimulus (compound), whereas the other auditory stimulus continued to be presented alone.

The data of primary interest come from the final extinction day and subsequent tests for spontaneous recovery. As shown in Figure $1 \mathrm{~B}$, on the final extinction day responding in the presence of the single stimulus (Y) continued to be low, whereas responding to the compound (AX) was higher. Notably, this pattern reversed when the stimulus that had been part of the compound was compared with the stimulus extinguished alone in tests conducted 1 wk or 4 wk later. This description is confirmed by the statistical analyses which revealed a significant effect of experimental phase (extinction, 1 -wk test, 4 -wk test; $F_{(2,22)}=52.3, P<0.01$ ), and while there was no overall effect of stimulus (compound vs. single; $\left.F_{(1,11)}=0.22, P>0.05\right)$ there was an interaction between stimulus and phase $\left(F_{(2,22)}=23.6, P<0.01\right)$. To further explore this interaction we compared responding to the two stimuli in each phase. During extinction responding was significantly higher for the compound $\left(F_{(1,11)}=86.6, P<0.01\right)$. In the 1 -wk test responding was low for both stimuli; however, consistent with improved extinction, responding was reduced for the stimulus that had been extinguished as part of a compound compared with the stimulus that was only extinguished alone $\left(F_{(1,11)}=\right.$ 8.1, $P<0.05)$. When the rats were tested $4 \mathrm{wk}$ later, this effect was more pronounced. While there was recovery of responding to both stimuli relative to extinction levels, the rats responded significantly more in the presence of the stimulus that had been extinguished alone compared with the stimulus that had been extinguished in compound, suggesting that the difference seen at $1 \mathrm{wk}$ was maintained $4 \mathrm{wk}$ after extinction $\left(F_{(1,11)}=7.3, P<\right.$ $0.01)$.

Because this effect was more robust at the 4-wk time point, this time point was used in subsequent experiments. These results replicate Rescorla's (2006) demonstration that extinguishing a compound reduced subsequent spontaneous recovery compared with a stimulus that was only extinguished alone at the 1-wk time point and further demonstrate that this difference is maintained for at least $4 \mathrm{wk}$.

Table 1. Summary of experimental design for Experiment 1

\begin{tabular}{lccc}
\hline Acquisition & Extinction 1 & Extinction 2 & Test \\
\hline $\mathrm{A}+$ & $\mathrm{A}-$ & $\mathrm{AX}-$ & $\mathrm{X} ?$ \\
$\mathrm{X}+$ & $\mathrm{X}-$ & $\mathrm{Y}-$ & $\mathrm{Y} ?$ \\
$\mathrm{Y}+$ & $\mathrm{Y}-$ & & \\
\hline
\end{tabular}

Summary of the experimental design used in Experiment 1. " $\mathrm{A}$ " represents the light stimulus. " $\mathrm{X}$ " and " $\mathrm{Y}$ " represent the noise and clicker stimuli (counterbalanced). " + " indicates that responding in the presence of the stimuli was rewarded, whereas " -" indicates extinction conditions. 

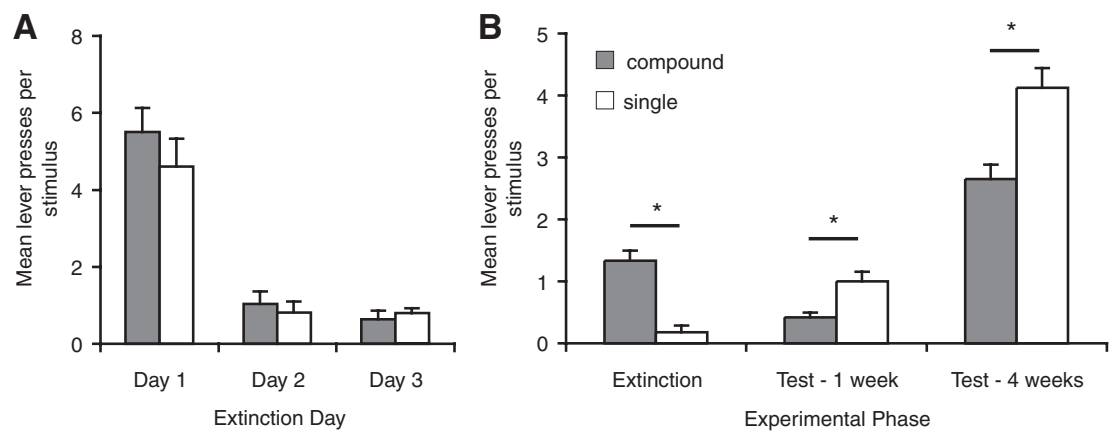

Figure 1. Extinction of a stimulus compound reduces spontaneous recovery relative to extinction of a single stimulus. $(A)$ Mean lever presses per stimulus for the initial extinction sessions. (B) Mean lever presses per stimulus on the final extinction session and in the tests conducted 1 and 4 wk later. Presentation of a stimulus compound increased responding during extinction; however, when the element of that compound was tested alone $1 \mathrm{wk}$ or $4 \mathrm{wk}$ later, animals responded less to the stimulus that was extinguished in compound than to the stimulus extinguished alone. Analysis revealed a significant effect of experimental phase indicating that responding was elevated at the 4-wk time point $\left(F_{(2,22)}=52.3, P<0.01\right)$. Further, while there was no effect of stimulus $\left(F_{(1,11)}=0.2, P>0.05\right)$, there was a significant interaction between stimulus and phase, indicating that there was greater spontaneous recovery of the stimulus extinguished alone $\left(F_{(2,22)}=23.6, P<0.01\right)$. ${ }^{*}$ Indicates a significant difference in number of lever presses; $P<0.05 ; N=12$.

\section{Experiment 2: Yohimbine treatment during extinction reduces spontaneous recovery}

While yohimbine, an $\alpha 2$ antagonist that increases synaptic NE, at high doses can produce a stress response sufficient to produce reinstatement (e.g., Ghitza et al. 2006), yohimbine has also been shown to promote extinction of conditioned fear (Cain et al. 2004; Morris and Bouton 2007; Powers et al. 2009; but see also Mueller et al. 2009). Specifically, when injected with yohimbine prior to presentation of a shock-predictive CS in extinction, mice showed a reduced fear response to the CS when tested drugfree the following day relative to saline controls. Additionally, while control mice did show extinction given enough trials, those treated with yohimbine showed extinction after fewer nonreinforced trials (Cain et al. 2004). The fact that a potentially anxiogenic drug facilitates extinction of conditioned fear is consistent with the demonstration that increased excitation, or arousal, during extinction leads to greater extinction (Rescorla 2000, 2006). The following experiment tested the hypothesis that increased NE activation produced by giving yohimbine during extinction learning will mimic the effects of compound stimulus presentation and enhance extinction of reward-predictive stimuli.

Rats were trained using the same discriminative stimulus procedure and underwent two sessions of initial extinction training as above. Prior to a third extinction session the rats were divided into groups in an attempt to match their training and initial extinction response rates and received an injection of either saline or 1.25 or $2.5 \mathrm{mg} / \mathrm{kg}$ yohimbine 30 min prior to session onset. Our prediction was that yohimbine treatment would mimic the effects of compound stimulus extinction and augment extinction learning; thus, the rats received further extinction of a single stimulus. Animals were tested 4 wk later for spontaneous recovery of responding.
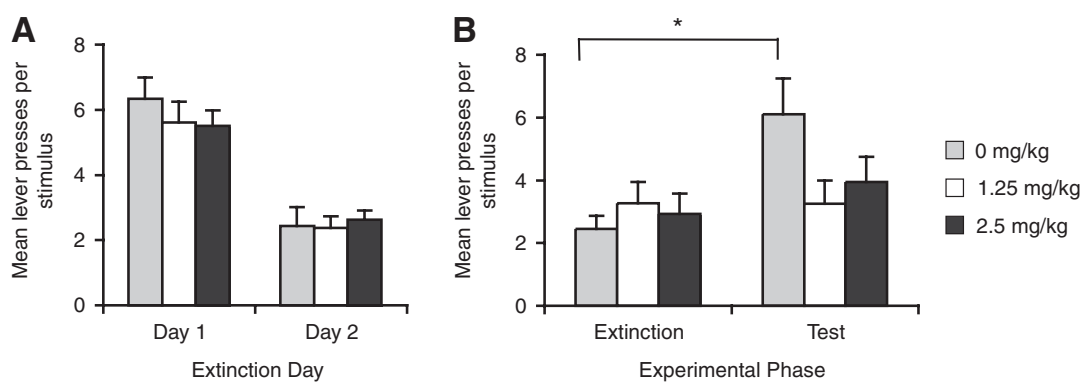

Figure 2. Yohimbine treatment enhances extinction. (A) Mean lever presses per stimulus for the initial extinction sessions. $(B)$ Mean lever presses per stimulus during the final extinction session containing the drug treatments and during the test session conducted drug-free $4 \mathrm{wk}$ later. Treatment with yohimbine during extinction had no effect on responding $\left(F_{(2,27)}<1\right)$; however, when rats were tested for spontaneous recovery $4 \mathrm{wk}$ later, while rats previously treated with saline showed a significant increase in responding $\left(F_{(1,9)}=7.0, P<0.05\right)$, those treated with yohimbine did not $\left(1.25 \mathrm{mg} / \mathrm{kg} ; F_{(1,9)}=1.8\right.$, $\left.P>0.05 ; 2.5 \mathrm{mg} / \mathrm{kg} ; F_{(1,9)}=1.4, P>0.05\right)$, indicating that yohimbine treatment enhanced extinction of an appetitive stimulus. ${ }^{*}$ Indicates a significant difference in the number of lever presses; $P<0.05$. $N=10$ per group. 
and Quirk 2010). To further examine the role of NE signaling in extinction learning we examined the effects of the NE transporter blocker atomoxetine. Atomoxetine is a selective NE reuptake inhibitor and has dose-dependent beneficial effects on different forms of impulsivity and behavioral inhibition in rats (Robinson et al. 2008), monkeys (Seu et al. 2009), and humans (Chamberlain et al. 2006). Given our hypothesis that increasing the postsynaptic effect of NE should improve extinction, NE reuptake inhibitors such as atomoxetine should also be expected to improve extinction.

Rats received training and initial extinction identical to that described above until the drug treatments and final extinction session. Prior to a third extinction session the rats were divided into groups in an attempt to match their training and initial extinction response rates and received an injection of either saline or 0.3 or $1.0 \mathrm{mg} / \mathrm{kg}$ atomoxetine $45 \mathrm{~min}$ prior to the beginning of the session. Our prediction was that atomoxetine treatment would augment stimulus processing and extinction learning, and so the rats received further extinction of a single stimulus. Animals were tested four weeks later for spontaneous recovery of responding.

As shown in Figure 3B, and consistent with the findings above, animals treated with saline during extinction of a single stimulus showed spontaneous recovery of responding in the 4 -wk test. This effect differed for the groups treated with atomoxetine, and was reduced in particular for those treated with the $1.0 \mathrm{mg} / \mathrm{kg}$ dose. The statistical analyses indicate an effect of group $\left(F_{(2,33)}=4.56, P<0.05\right)$, of experimental phase (extinction vs. test; $\left.F_{(1,33)}=56.6, P<0.01\right)$ and an interaction between these factors $\left(F_{(2,33)}=3.25, P<0.05\right)$. Analysis of the difference in responding between extinction and test for each group confirmed an increase in responding between extinction and test for each group (saline; $F_{(1,12)}=17.9, P<0.01 ; 0.3 \mathrm{mg} / \mathrm{kg} ; F_{(1,9)}=19.6$, $\left.P<0.01 ; 1.0 \mathrm{mg} / \mathrm{kg} ; F_{(1,12)}=6.26, P<0.05\right)$; however, post-hoc analyses (Bonferroni) indicate that rats treated with $1.0 \mathrm{mg} / \mathrm{kg}$ of atomoxetine responded significantly less than those treated with saline.

\section{Experiment 3B: Learning vs. consolidation}

While the above results demonstrate that atomoxetine treatment can improve extinction, it remains unclear whether this is the result of increased stimulus processing or the result of improved consolidation of the extinction memory as NE has been implicated in both (McGaugh 2004; Arnsten 2009). In order to address the possibility that atomoxtine treatment specifically improves consolidation of extinction we compared the effects of pre- and post-training administration of the drug. Two groups of rats received $1.0 \mathrm{mg} / \mathrm{kg}$ of atomoxetine either prior to or immediately following the final extinction session. A third group received saline $(1 \mathrm{~mL} / \mathrm{kg})$ immediately following the session.

The extinction data from the final extinction session and test 4 wk later (panel D) are shown in Figure 3. As illustrated in the figure, we replicated our finding that pretraining atomoxetine reduces spontaneous recovery of responding providing evidence of enhanced extinction learning. The post-training treatment also reduced spontaneous recovery, although, to a lesser extent that the pretraining treatment. This description is confirmed by the statistical analysis which reveals an effect of experimental phase indicating that responding was higher at test than in extinction $\left(F_{(1,2)}=67.7, P<0.001\right)$. There was also an effect of group $\left(F_{(2,21)}=7.8, \quad P<\right.$ $0.01)$, but no interaction between these factors $\left(F_{(2,21)}=2.2, \quad P>0.05\right)$. Given the hypothesis that atomoxetine should reduce spontaneous recovery we predicted that the groups should differ specifically at test, and so we compared the groups in this session and found an effect of group $\left(F_{(2,21)}=7.6, P<0.01\right)$. Pairwise comparisons indicate that pretraining atomoxetine reduced spontaneous recovery relative to saline treatment $\left(t_{(12)}=4.1, P<0.001\right)$. Post-training treatment also reduced spontaneous recovery compared with saline $\left(t_{(15)}=2.1, P<\right.$ $0.05)$. However, the pretraining and posttraining groups also differed from each other $\left(t_{(15)}=2.1, P<0.05\right)$ with the posttraining group responding more at test 
than the pretraining group, suggesting that consolidation may contribute to the effect seen with atomoxetine but that this probably does not explain the entire effect. If we impose the more conservative Bonferroni correction, only the pretraining treatment differs from saline. An effect of the post-training treatment would be consistent with a role for NE in the consolidation of the extinction learning, and it is possible that different timing or some other parameter change may yield a greater effect of the post-training treatment. However, the fact that a greater effect was observed with pretraining treatment suggests that consolidation processes do not entirely explain the results, and that atomoxetine may also enhance stimulus processing or other attentive mechanisms during extinction learning.

\section{Experiment 4A: Propranolol treatment during extinction blocks the benefit to extinction seen with compound stimulus presentation}

While the effects of yohimbine or atomoxetine treatment shown above mimic the effects of extinguishing a stimulus compound, they do not prove that compound stimulus presentation activates NE signaling. $\beta$-Receptor activation has been shown to modulate many of the postsynaptic mechanisms involved in establishing long-term memories. For example, $\beta$-receptor agonists have been shown to facilitate synaptic transmission (Abraham et al. 2008; Pu et al. 2009) and stimulation of the locus coeruleus or local administration of the $\beta$-agonist isoproterenol paired with exposure to a novel odor were sufficient to condition a preference for that odor (Sullivan et al. 2000). Further, $\beta$-receptor antagonists such as propranolol block the beneficial effects that are typically seen with NE activation, for example, in response to emotional arousal (Cahill et al. 1994; De Martino et al. 2008), or exposure to a novel context (King and Williams 2009). In order to address whether NE signaling contributes to the effects seen with compound stimulus presentation, we tested the effects of blocking postsynaptic noradrenergic receptors on the efficacy of extinction by pretreating rats with the $\beta$-adrenergic receptor antagonist propranolol. If the effects of compound stimulus presentation depend on NE signaling, propranolol treatment should reduce the efficacy of this treatment and rats that were extinguished under propranolol should show increased spontaneous recovery.

Rats received training and initial extinction identical to that described above until the drug treatments and final extinction session. Our working hypothesis was that the compound stimulus presentation increases noradrenergic activity and reducing this effect (via blockade of $\beta$-receptors) should occlude the benefit to extinction. To test this hypothesis, separate groups of rats were given either $0,2.5$, or $5 \mathrm{mg} / \mathrm{kg}$ of propranolol prior to a further extinction of a stimulus compound (click or noise paired with light; balanced within groups). As shown in Figure 4, rats in the saline group showed robust responding to the stimulus compound but showed reduced responding to the element of that compound when tested 4 wk later. In contrast, treatment with propranolol reduced the impact of the compound stimulus presentation during extinction (i.e., rats performed fewer responses) and, importantly, rats treated with propranolol exhibited greater spontaneous recovery when the element was subsequently tested. The statistical analyses revealed no overall effect of group $\left(F_{(2,36)}=0.22, P>0.05\right)$, but there was an effect of experimental phase $\left(F_{(1,36)}=12.4, P<0.01\right)$ and an interaction between these factors $\left(F_{(2,36)}=7.8, P<0.01\right)$. Because the rats received a stimulus compound in the extinction session but a single stimulus in the test session the amount of responding between these sessions is expected to differ; therefore, in order to further evaluate the drug effects, we focused on between-, rather than within-group comparisons. Simple effects analyses demonstrate that treatment with propranolol reduced responding during the extinction phase $\left(F_{(2,36)}=3.9, P<0.05\right)$, and post-hoc analyses (Bonferroni) indicate that rats treated with $5.0 \mathrm{mg} / \mathrm{kg}$ responded less than those treated with saline, but that rats treated with $2.5 \mathrm{mg} / \mathrm{kg}$ did not differ from the other two doses. During the test phase, the effect of group was marginal $\left(F_{(2,36)}=2.9, P=\right.$ 0.07); however, post-hoc analyses indicate that responding
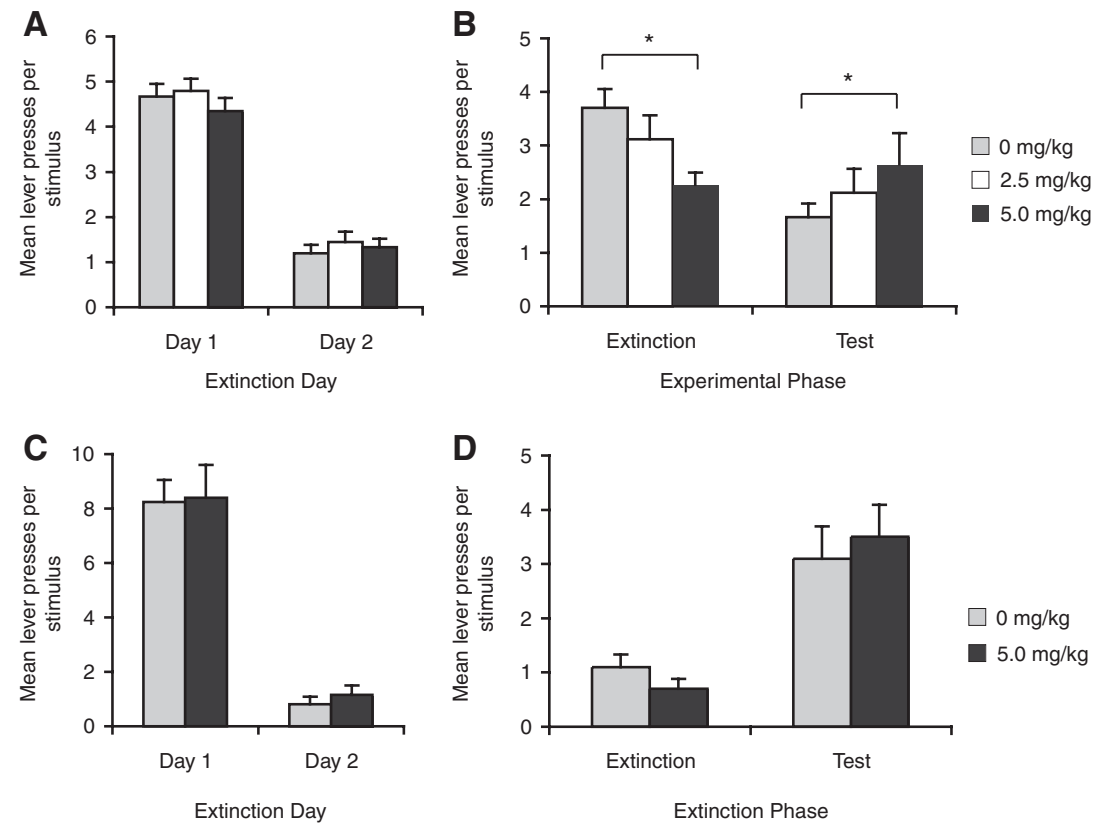

Figure 4. Propranolol treatment blocks the benefit to extinction produced by extinction of a stimulus compound. (A) Mean lever presses per stimulus for the initial extinction sessions for Experiment 4A. (B) Mean lever presses per stimulus during the final extinction session containing the drug treatments and during the test session conducted drug-free $4 \mathrm{wk}$ later. In saline-treated animals compound stimulus presentation produced robust responding in extinction and reduced responding when the element was tested 4 wk later. In contrast, the group treated with $5.0 \mathrm{mg} / \mathrm{kg}$ of propranolol showed less responding in extinction when the drug was present but greater spontaneous recovery when subsequently tested drug-free. Analysis revealed no effect of group $\left(F_{(2,36)}=0.22, P>0.05\right)$, but there was an effect of experimental phase $\left(F_{(1,36)}=12.4, P<0.01\right)$ and an interaction between these factors $\left(F_{(2,36)}=7.8, P<0.01\right)$. Post-hoc analyses indicated that rats treated with $5.0 \mathrm{mg} / \mathrm{kg}$ of propranolol responded less in test than those treated with saline. *Indicates $P<0.05$ for post-hoc analyses. These results suggest that propranolol occludes the benefit to extinction otherwise achieved with compound stimulus presentation. $N=14,13$, and 12 for the $0,2.5$, and $5.0 \mathrm{mg} / \mathrm{kg}$ groups, respectively. (C) Mean lever presses per stimulus for the initial extinction sessions for Experiment 4B. (D) Propranol treatment was without effect on extinction of a single stimulus. Analysis revealed an effect of experimental phase, indicating that both groups increased responding from extinction to test $\left(F_{(1,16)}=34.8, P<0.01\right)$; there was no effect of group $(F<1)$, and no interaction between these factors $\left(F_{(1,16)}=2.5, P>0.05\right) . N=9$ per group. 
was greater for rats treated with the high dose of propranolol than for those treated with saline indicating greater spontaneous recovery in this group.

\section{Experiment 4B: Propranolol does not affect extinction of a single stimulus}

It remains possible that propranolol treatment interferes with extinction in general rather than specifically blocking the enhancing effect of the compound presentation. To address this possibility we tested two more groups of rats that received either saline or propranolol $(5.0 \mathrm{mg} / \mathrm{kg})$ prior to a final extinction session containing presentations of a single stimulus. The extinction data (panel C) and data from the final extinction session and test (panel D) are shown in Figure 4. As suggested by the figure, propranolol given with extinction of a single stimulus had no effect on spontaneous recovery tested $4 \mathrm{wk}$ later. While there was an effect of experimental phase, indicating that both groups increased responding from extinction to test $\left(F_{(1,16)}=34.8, P<0.01\right)$, there was no effect of group $(F<1)$, and no interaction between these factors $\left(F_{(1,16)}=2.5, P>0.05\right)$.

These data indicate that propranolol does not produce a general impairment in extinction but its effects in Experiment $4 \mathrm{~A}$ are specific to the compound stimulus. These results further suggest that the effects of the compound stimulus depend, at least in part, on noradrenergic activity during extinction training and that this benefit to extinction can be reduced by propranolol.

\section{Experiment 5: Control for nonspecific effects of drug treatment}

Of note, propranolol treatment during exposure to the stimulus compound reduced the amount of responding compared with animals treated with saline. Thus, it is possible that the fact that these animals responded less during the session resulted in reduced extinction. In order to address this possibility we conducted a further experiment to examine any nonspecific effects of drug treatment (e.g., related to response performance, context exposure, etc.). Rats underwent training and initial extinction (two sessions with stimuli present) identical to that described above. Prior to the third extinction session rats were divided into four groups and received either saline, yohimbine $(2.5 \mathrm{mg} / \mathrm{kg})$, atomoxetine $(1.0 \mathrm{mg} / \mathrm{kg})$, or propranolol $(5.0 \mathrm{mg} /$ $\mathrm{kg}$ ) prior to an additional extinction session where the levers were present but no stimuli were presented.

As shown in Figure 5, rats showed similar low levels of responding following the drug treatment. Further, when tested 4 wk later, all groups showed similar levels of spontaneous recovery. This description is confirmed by the statistical analyses which indicate an effect of experimental phase $\left(F_{(1,28)}=\right.$ 103.8, $P<0.01)$ but no effect of group $\left(F_{(3,28)}=0.4, P>0.05\right)$ and no interaction between these factors $\left(F_{(3,28)}=\right.$ $0.1, \quad P>0.05)$. These data suggest that the effects of noradrenergic drugs reported above are related to stimulus exposure and extinction. While not entirely surprising given that the rats were trained that only responding in the presence of the stimuli resulted in reward, nonetheless, these results help rule out factors such as motor impairments or state-specific learning effects which could contribute to the results reported above.

\section{Discussion}

These data replicate the previous demonstration by Rescorla (2006) that presenting a stimulus compound in extinction augments the extinction of the elements of that compound relative to a stimulus that was only extinguished alone. In addition, we find that this effect is long lasting, with significant effects on recovery of responding that last at least $4 \mathrm{wk}$. Further, the results from Experiment 2 extend findings from fear-conditioning paradigms (e.g., Cain et al. 2004) and provide additional evidence that the $\alpha-2$ noradrenergic antagonist, yohimbine, can enhance the efficacy of extinction training, making it is less vulnerable to later disruption, such as that which occurs following the passage of time. Similar results found with the NE uptake inhibitor, atomoxetine, suggest that these effects are due to increased noradrenergic activity rather than other off target effects of yohimbine. While the effects of yohimbine or atomoxetine treatment mimic the effects of compound stimulus presentation on extinction learning, the results of Experiment 4 showing that the noradrenergic $\beta$-antagonist, propranolol, blocks the benefit to extinction otherwise seen with compound stimulus presentation provide the most direct evidence that compound stimulus presentation recruits noradrenergic activity. Together, these results demonstrate that compound stimulus presentation can improve extinction learning, and that this effect may be mediated by activation of NE. The effects of compound stimulus manipulation are readily interpretable in terms of increased prediction error resulting from the presentation of multiple predictors of reward. While NE activity may not increase prediction errors per se, the current results demonstrate that manipulations that increase activity of the NE system can likewise promote learning in extinction.

\section{Receptor specificity}

These experiments were conducted with the overall hypothesis that increasing noradrenergic activity would improve, whereas decreasing noradrenergic activity would impair extinction learning. NE function involves actions distributed across multiple
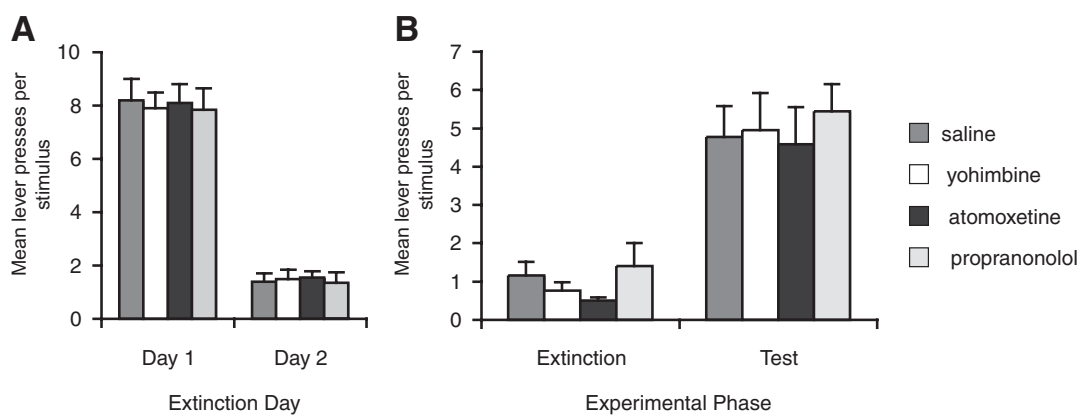

Figure 5. Drug treatment without stimulus exposure does not alter responding at test. $(A)$ Mean lever presses per minute during the extinction session containing the drug treatments and during the test conducted drug-free $4 \mathrm{wk}$ later. Note that in the final extinction session (presented above) the levers were present but the stimuli withheld. When animals were given drug treatments without exposure to the discriminative stimuli, there was no effect on response rates either during extinction or when the stimuli were reintroduced in the test. $(B)$ Statistical analyses indicate an effect of experimental phase $\left(F_{(1,28)}=103.8, P<0.01\right)$ but no effect of group $\left(F_{(3,28)}=0.4, P>0.05\right)$ and no interaction between these factors $\left(F_{(3,28)}=0.1, P>0.05\right)$. These data suggest that the effects of noradrenergic drugs reported above are related to stimulus exposure and extinction. $N=8$ per group. 
anatomical regions and multiple receptor subtypes, not all of which were tested here. While the current results implicate antagonism of the $\alpha 2$-receptor or administration of NET inhibitors as a means for increasing $\mathrm{NE}$, and antagonism of $\beta$ receptors as a means for decreasing the postsynaptic effects of NE, other subclasses of receptors may also play important roles in mediating appetitive extinction. For example, recent work has demonstrated that either pretraining (Do-Monte et al. 2010) or post-training (Bernardi and Lattal 2010) administration of the $\alpha 1$ antagonist prazosin slows extinction of conditioned fear implicating the $\alpha 1$ receptor in extinction. The role of the $\alpha 1$-receptor in the current paradigm awaits further testing.

\section{Increased responding vs. increased extinction}

It is interesting to note that in some of the experiments above, the amount of responding during extinction is negatively correlated with the amount of spontaneous recovery observed at test. For example, increased responding to the compound corresponds to less responding at test and less responding following propranolol treatment corresponds to more responding at test. Thus, it is plausible that the amount of responding rather than attentional or associative mechanisms accounts for the differences observed. Notably, these relations did not always occur, i.e., treatment with either yohimbine or atomoxetine did not increase responding during extinction but did reduce responding at test. Further, this issue has been directly assessed in an experiment by Rescorla (2006) (Experiment 5). There, using a sign-tracking procedure, he demonstrated that presentation of a stimulus trained as a facilitator, that itself had no net excitatory strength but was nonetheless able to augment responding to another trained excitor when they were presented together, failed to promote extinction. In contrast, presentations of a diffuse excitor, a stimulus that produced a conditioned response distinct from that produced by the target stimulus that thus failed to increase responding when the stimuli were presented together, did facilitate extinction. These results are consistent with an error prediction account of the augmented extinction rather than an account based on elevated response performance alone. Further, our own Experiment 5 was conducted to examine any effects of the drug treatments that were not related to stimulus presentation (e.g., context exposure, effects on the lever-press response). We found that the drug treatments were without effect on spontaneous recovery if the rats were not given exposure to the stimuli. These data suggest that changes in the amount of responding alone are not sufficient to promote or interfere with extinction learning, and that the effects of the drug treatments in these experiments are specific to the stimuli.

\section{Improved extinction vs. disruption of reconsolidation}

Post-training administration of noradrenergic compounds can modulate retention of the preceding training leading to the theory that NE is important for memory consolidation (for review, see McGaugh 2004). Several studies examining the effects of noradrenergic compounds on extinction learning have used pretraining treatments (Cain et al. 2004; Morris and Bouton 2007) where the effects on acquisition of extinction vs. disruption of consolidation or reconsolidation of the original excitatory memory are difficult to disentangle. In Experiment 3 we examined the effects of post-training administration of atomoxetine and saw a decrease in spontaneous recovery that was less robust than that seen with the pretraining treatment. It remains possible that different parameters or drugs working through different mechanisms (including yohimbine) may yield different results and so the current results observed mainly following pretraining treatments do not rule out a role of NE in consolidation of an extinction memory. This important distinction between the contribution of NE to acquisition of extinction vs. consolidation of the extinction memory will be addressed in future studies.

\section{Contextual modulation}

It is well established that the expression of extinction can depend on the context in which it is tested (Bouton and Bolles 1979; Bouton 2004). For example, rats that are trained in one context but extinguished in another will show renewal of responding when returned to the original training context. Indeed, using a fear-conditioning paradigm, it has been shown that administration of noradrenergic agents, such as yohimbine, while facilitating extinction of conditioned fear, can interact with the context where either extinction occurs, or the drug is administered (Morris and Bouton 2007). As such, it is possible that the effects reported here may be altered if rats are tested outside the environment in which they were extinguished. The role of contextual control of extinction learning and its mediation by NE, while not specifically addressed in the experiments above, is an important area for future study.

\section{Clinical implications}

Extinction-based therapies have been used for the treatment of anxiety disorders and phobias as well as substance abuse where they have met with some success (O'Brien et al. 1990; Gould et al. 1995; Pollack et al. 2002; Otto et al. 2004; Craske et al. 2008). Nonetheless, they are not always effective (e.g., Marissen et al. 2007). However, new developments in our understanding of extinction itself, developed largely from animal studies, suggest that this strategy warrants further investigation (Bouton 2002; Conklin and Tiffany 2002; Chaudhri et al. 2008; Taylor et al. 2009). There have been some recent significant advances in the efficacy of extinction-based therapies driven by insights from animal studies and resulting from both improvements in the behavioral methods and the use of pharmacological adjuncts (Craske et al. 2008; Norberg et al. 2008). The addition of pharmacological agents within the clinic setting may be able to safely augment the extinction learning that occurs as a valuable addition to cognitive-behavioral-based therapies to ultimately improve the efficacy of this treatment. Equally important, a better understanding of the pharmacological regulation of extinction learning may identify commonly prescribed drugs that could interfere with extinction and be counterproductive to other therapeutic interventions.

\section{Conclusions}

In summary, we find that compound stimulus presentation during extinction results in a long-lasting enhancement of that extinction. Extinction is likewise enhanced by drugs that increase noradrenergic activity and the benefit of compound stimulus presentation is blocked by a noradrenergic $\beta$-antagonist. Taken in the broader context of learning, the noradrenergic system has been shown to have powerful effects on neural responses and consequent synaptic plasticity associated with regulating long-term memory formation (Yang et al. 1996; Berridge and Waterhouse 2003; Sara 2009). The current results indicate that noradrenergic effects on learning include the special case of extinction and may help improve clinical applications. 


\section{Materials and Methods}

\section{Experiment 1}

\section{Subjects and apparatus}

The subjects were 12 male Sprague-Dawley rats (Charles River Laboratory, Hollister, CA) approximately $300 \mathrm{~g}$ at the beginning of the experiment. They were housed in individual cages and their feeding was restricted such that they were maintained at approximately $90 \%$ of their free-feeding weight for the duration of the experiment. They had free access to water while in the home cage. All procedures were approved by the Institutional Animal Care and Use Committee of the Ernest Gallo Clinic and Research Center at the University of California, San Francisco. Training and testing took place in Med Associates operant chambers housed within sound- and light-attenuating shells. Each chamber was equipped with a pellet dispenser that delivered a single 45-mg pellet (BioServ) into a recessed magazine in the chamber when activated. The chambers contained retractable levers that could be inserted to the left and right of the magazine. The boxes also contained two white key lights (one over each lever), a white noise generator, and a solanoid that, when activated, delivered a $5-\mathrm{Hz}$ clicker stimulus. The auditory stimuli were adjusted to $80 \mathrm{~dB}$ in the presence of background noise of $60 \mathrm{~dB}$ provided by a ventilation fan. A $3-\mathrm{W}, 24-\mathrm{V}$ house light mounted on the top center of the wall opposite the levers and magazine provided illumination. Computers equipped with MED-PC software controlled the equipment and recorded magazine entries and lever-press responses.

\section{Procedure}

\section{Magazine and response training}

These procedures were designed after those of Rescorla (2006) (Experiment 3). The rats were gradually food restricted and handled daily for $1 \mathrm{wk}$ prior to the beginning of the experiment. On the first training day the rats received a 30-min magazine training session during which approximately 30 pellets were delivered on a random time 60 -sec schedule. The levers were retracted for this session. On the next day, the rats were trained to press the lever (left or right, balanced across subjects) for a food pellet. Each lever press delivered a pellet until 50 pellets were earned, at which point the session was terminated. Rats that failed to earn 50 pellets in the initial session were given a second session the following day.

\section{Acquisition}

The next day discrimination training began. Each session contained eight 30-sec presentations each of a light (two key lights illuminated), white noise, and clicker stimulus (24 trials total). During each stimulus, lever pressing resulted in pellet delivery. In order to aid acquisition, on the first day the lever was inserted at the beginning and retracted at the end of each stimulus. On subsequent days the lever was present throughout the session but only responding during the stimuli was reinforced according to a random ratio (RR) schedule that was increased across days (days 1-3: RR1; days 4-6: RR2; days 7-9: RR4). Trial order was determined by the computer program and the intertrial interval (ITI) was variable but on average was $90 \mathrm{sec}$. The rats received a total of nine reinforced training sessions with the discriminative stimuli.

\section{Extinction phase 1}

On the following $2 \mathrm{~d}$ the rats received sessions identical to the training sessions except that no pellets were delivered.

\section{Extinction phase 2}

On the following day, the rats received 12 single stimulus trials (four of each stimulus) followed by six further nonreinforced presentations of each of two trial types, AX- and Y-. The two auditory stimuli served as stimuli $\mathrm{X}$ and $\mathrm{Y}$ in a counterbalanced fashion and the light stimulus served as stimulus $\mathrm{A}$. The entire session had 24 trials as in previous sessions and each stimulus had an equal number of presentations.

\section{Test}

The rats remained in their home cages for $1 \mathrm{wk}$ maintained on their deprivation schedule before testing. The test session contained four presentations of each the noise and clicker stimuli in pseudorandom order. The rats received a second test session identical to the first conducted $4 \mathrm{wk}$ after the final extinction session.

\section{Experiment 2}

\section{Subjects and apparatus}

The subjects were 30 male Sprague-Dawley rats maintained in the same manner as those from Experiment 1 above. The apparatus was identical to that used in the previous experiment.

\section{Procedure}

The initial training including magazine training, acquisition, and extinction phase 1 were identical to the description in Experiment 1 . The rats received a saline injection prior to the final rewarded training session in order to habituate them to the injection procedure.

\section{Extinction phase 2}

Based on their responding in the previous extinction the rats were divided into three groups with approximately even response rates. On the following day, rats received an injection of either saline or 1.25 or $2.5 \mathrm{mg} / \mathrm{kg}$ yohimbine (yohimbine hydrochloride; Sigma; dissolved in saline and administered in a volume of $1 \mathrm{~mL} / \mathrm{kg}$ ) $30 \mathrm{~min}$ prior to the beginning of the session. Our prediction was that yohimbine treatment would augment stimulus processing and extinction learning, and so the rats received further extinction of a single stimulus (six trials; clicker or noise balanced within groups and total responding for the assigned stimulus in previous extinction sessions was approximately equated across groups).

\section{Test}

Animals were tested $4 \mathrm{wk}$ later for spontaneous recovery of responding. The test consisted of six presentations of the same stimulus that was presented in the final extinction session and the number of lever-press responses were recorded. No food pellets were delivered in this session.

\section{Experiment 3}

\section{Procedure}

Experiment $3 A$. Thirty-six rats were housed and trained, and divided into groups in an identical fashion to that described above until the drug treatment day. Rats received an injection of either saline, 0.3 or $1.0 \mathrm{mg} / \mathrm{kg}$ atomoxetine (atomoxetine hydrochloride; Tocris; dissolved in saline and administered in a volume of $1 \mathrm{~mL} / \mathrm{kg}$ ) $45 \mathrm{~min}$ prior to the beginning of the session.

Experiment 3B. Twenty-four male Sprague-Dawley rats (ARC) weighing approximately $300 \mathrm{~g}$ at the beginning of the experiment served as subjects. They were housed in squads of eight and their feeding was restricted such that they were maintained at approximately $90 \%$ of their free-feeding weight for the duration of the experiment. They had free access to water while in the home cage. All procedures were approved by the Institutional Research Ethics Committee of the University of Sydney. Training was conduced in Med-Associates operant chambers outfitted as those described above. Rats were trained and divided into groups as above and received $1.0 \mathrm{mg} / \mathrm{kg}$ atomoxetine either $45 \mathrm{~min}$ prior 
to the beginning of the session or immediately upon completion of the session or sterile saline immediately after the session.

Our prediction was that atomoxetine treatment would augment stimulus processing and extinction learning, and so the rats received further extinction of a single stimulus as above.

Test

Animals were tested 4 wk later for spontaneous recovery of responding following the procedures of Experiment 2 above.

\section{Experiment 4}

\section{Procedure}

Experiment $4 A$. Thirty-nine male rats were maintained and trained in an identical fashion to Experiment 1 until the drug treatment and final extinction session. Our working hypothesis was that the compound stimulus presentation increases noradrenergic activity and reducing this effect (via blockade of $\beta$-receptors) would occlude the benefit to extinction. To test this hypothesis, groups of rats were given either $0,2.5$, or $5 \mathrm{mg} / \mathrm{kg}$ of propranolol (propranolol hydrochloride; Sigma; dissolved in sterile water) 30 min prior to a final extinction session containing six presentations of a stimulus compound (click or noise paired with light; balanced within groups). Animals were tested $4 \mathrm{wk}$ later for spontaneous recovery of responding.

Experiment $4 B$. Eighteen rats were maintained and trained in an identical fashion to Experiment 3B until the drug treatment. To address whether propranolol affects extinction, generally the rats were divided into groups and received either 0 or $5 \mathrm{mg} / \mathrm{kg}$ of propranolol prior to a final extinction session containing six single stimulus trials. Animals were tested 4 wk later for spontaneous recovery of responding.

\section{Experiment 5}

\section{Procedure}

Thirty-two rats were maintained and trained in an identical fashion to that described in the experiments above. In order to control for any nonspecific effects of the drug treatments such as motor impairment, sedation, state-dependent learning, and so on, we examined the effects of drug treatment prior to a session in which the rats were exposed to the chambers and had the opportunity to perform the lever-press response; however, the stimuli were not presented. Prior to this session rats were divided into groups based on their response rates in the first two extinction sessions and treated with either saline, yohimbine $(2.5 \mathrm{mg} /$ $\mathrm{kg})$, atomoxetine $(1.0 \mathrm{mg} / \mathrm{kg})$, or propranolol $(5.0 \mathrm{mg} / \mathrm{kg})$. These doses were chosen as they were the doses found to have the clearest effect on spontaneous recovery in the experiments above. The session was $20 \mathrm{~min}$ in duration which was the approximate duration of the sessions containing stimuli in the other experiments. The rats were tested 4 wk later for spontaneous recovery of responding. The stimuli were again present in this test.

\section{Statistics}

Data were analyzed with repeated-measures analysis of variance (ANOVA) and simple effects analyses and Bonferroni post-hoc tests were used to further assess main effects and interactions where indicated.

\section{Acknowledgments}

The work was supported by a grant from the National Institute of Drug Abuse to P.H.J. (215408) and funds from the University of Sydney School of Psychology to L.H.C.

\section{References}

Abraham PA, Xing G, Zhang L, Yu EZ, Post R, Gamble EH, Li H. 2008. Beta1and beta 2 adrenoceptor induced synaptic facilitation in rat basolateral amygdala. Brain Res 1209: 65-73.

Arnsten AF. 2009. Stress signalling pathways that impair prefrontal cortex structure and function. Nat Rev Neurosci 10: $410-422$.

Berlau DJ, McGaugh JL. 2006. Enhancement of extinction memory consolidation: The role of the noradrenergic and GABAergic systems within the basolateral amygdala. Neurobiol Learn Mem 86: 123-132.

Bernardi RE, Lattal KM. 2010. A role for $\alpha 1$-adrenergic receptors in extinction of conditioned fear and cocaine conditioned place preference. Behav Neurosci 124: 204-210.

Berridge CW, Waterhouse BD. 2003. The locus coeruleus-noradrenergic system: Modulation of behavioral state and state-dependent cognitive processes. Brain Res Rev 42: 33-84.

Bouton ME. 2002. Context, ambiguity, and unlearning: Sources of relapse after behavioral extinction. Biol Psychiatry 52: 976-986.

Bouton ME. 2004. Context and behavioral processes in extinction. Learn Mem 11: 485-494.

Bouton ME, Bolles RC. 1979. Role of conditioned contextual stimuli in reinstatement of extinguished fear. J Exp Psychol Anim Behav Process 5: $368-378$

Bouton ME, King DA. 1983. Contextual control of the extinction of conditioned fear: Tests for the associative value of the context. J Exp Psychol Anim Behav Process 9: 248-265.

Cahill L, Prins B, Weber M, McGaugh JL. 1994. Beta-adrenergic activation and memory for emotional events. Nature 371: 702-704.

Cain CK, Blouin AM, Barad M. 2004. Adrenergic transmission facilitates extinction of conditioned fear in mice. Learn Mem 11: 179-187.

Chamberlain SR, Müller U, Blackwell AD, Clark L, Robbins TW, Sahakian BJ. 2006. Neurochemical modulation of response inhibition and probabilistic learning in humans. Science 311: 861-863.

Chaudhri N, Sahuque LL, Janak PH. 2008. Context-induced relapse of conditioned behavioral responding to ethanol cues in rats. Biol Psychiatry 64: 203-210.

Conklin CA, Tiffany ST. 2002. Applying extinction research and theory to cue-exposure addiction treatments. Addiction 97: 155-167.

Craske MG, Kircanski K, Zelikowsky M, Mystkowski J, Chowdhury N, Baker A. 2008. Optimizing inhibitory learning during exposure therapy. Behav Res Ther 46: 5-27.

Delamater AR. 1996. Effects of several extinction treatments upon the integrity of Pavlovian stimulus-outcome associationa. Anim Learn Behav 24: 437-449.

De Martino B, Strange BA, Dolan RJ. 2008. Noradrenergic neuromodulation of human attention for emotional and neutral stimuli. Psychopharmacology (Berl) 197: 127-136.

Dolan RJ. 2002. Emotion, cognition, and behavior. Science 298: $1191-1194$.

Do-Monte FHM, Allensworth M, Carobrez AP. 2010. Impairment of contextual conditioned fear extinction after microinjection of alpha-1-adrenergic blocker prazosin into the medial prefrontal cortex. Behav Brain Res 211: 89-95.

Ghitza UE, Gray SM, Epstein DH, Rice KC, Shaham Y. 2006. The anxiogenic drug yohimbine reinstates palatable food seeking in a rat. Neuropsychopharmacology 31: 2188-2196.

Gould R, Otto MW, Pollack MH. 1995. A meta-analysis of treatment outcome for panic disorder. Clin Psychol Rev 15: 819-844.

Holmes A, Quirk GJ. 2010. Pharmacological facilitation of fear extinction and the search for adjunct treatments for anxiety disorders-the case of yohimbine. Trends Pharmacol Sci 31: 2-7.

Horns HL, Heron WR. 1940. A study of disinhibition in the white rat. J Comp Psychol 30: 97-102.

Kamin LJ. 1968. "Attention-like" processes in classical conditioning. In Miami Symposium on the prediction of behavior: Aversive stimulation (ed. MR Jones), pp. 9-31. University of Miami Press, Miami, FL.

King SO, Williams C. 2009. Novelty-induced arousal enhances memory for cued classical fear conditioning: Interactions between peripheral adrenergic and brainstem glutamatergic systems. Learn Mem 16: 625-634.

Marissen MA, Franken IH, Blanken P, van den Brink W, Hendriks VM. 2007. Cue exposure therapy for the treatment of opiate addiction: Results of a randomized controlled clinical trial. Psychother Psychosom 76: $97-105$.

McGaugh JL. 2004. The amygdala modulates the consolidation of memories of emotionally arousing experiences. Annu Rev Neurosci 27: $1-28$.

Morris RW, Bouton ME. 2007. The effect of yohimbine on the extinction of conditioned fear: A role for context. Behav Neurosci 121: 501-514.

Mueller D, Olivera-Figueroa LA, Pine DS, Quirk GJ. 2009. The effects of yohimbine and amphetamine on fear expression and extinction in rats. Psychopharmacology (Berl) 204: 599-606. 
Norberg MM, Krystal JH, Tonlin DF. 2008. A meta-analysis of D-cycloserine and the facilitation of fear extinction and exposure therapy. Biol Psychiatry 63: 118-126.

O'Brien CP, Childress AR, McLellan T, Ehrman R. 1990. Integrating systematic cue exposure with standard treatment in recovering drug dependent patients. Addict Behav 15: 355-365.

O'Carroll RE, Drysdale E, Cahill L, Shajahan P, Ebmeier KP. 1999. Stimulation of the noradrenergic system enhances and blockade reduces memory for emotional material in man. Psychol Med 29: $1083-1088$.

Otto MW, Safren SA, Pollack MH. 2004. Internal cue exposure and the treatment of substance use disorders: Lessons from the treatment of panic disorder. J Anxiety Disord 18: 69-87.

Pavlov IP. 1927. Conditioned reflexes. Oxford University Press, Oxford, UK.

Pollack MH, Penava SA, Bolton E, Worthington JJ 3rd, Allen GL, Farach FJ Jr, Otto MW. 2002. A novel cognitive-behavioral approach for treatment-resistant drug dependence. J Subst Abuse Treat 23: $335-342$.

Powers MB, Smits JAJ, Otto MW, Sanders C, Emmelkamp PMG. 2009. Facilitation of fear extinction in phobic participants with a novel cognitive enhancer: A randomized placebo controlled trial of yohimbine augmentation. J Anxiety Disord 23: 350-356.

Pu Z, Krugers HJ, Joëls M. 2009. Beta-adrenergic facilitation of synaptic plasticity in the rat basolateral amygdala in vitro is gradually reversed by corticosterone. Learn Mem 16: 155-160.

Reberg D. 1972. Compound tests for excitation in early acquisition and after prolonged extinction of conditioned suppression. Learn Motiv 3: 246-258.

Rescorla RA. 1996. Preservation of Pavlovian associations through extinction. QJ Exp Psychol 49B: 245-258.

Rescorla RA. 1997. Spontaneous recovery of instrumental discriminative responding. Anim Learn Behav 25: 99-107.

Rescorla RA. 2000. Extinction can be enhanced by a concurrent excitor. J Exp Psychol Anim Behav Process 26: 251-260.

Rescorla RA. 2006. Deepened extinction from compound stimulus presentation. J Exp Psychol Anim Behav Process 32: 135-144.

Rescorla RA, Heth CD. 1975. Reinstatement of fear to an extinguished conditioned stimulus. J Exp Psychol Anim Behav Process 1: 88-96.

Rescorla RA, Wagner AR. 1972. A theory of Pavlovian conditioning: Variations in the effectiveness of reinforcement and nonreinforcement. In Classical conditioning II: Current research and theory (ed. AHP Black, WF Prokasy), pp. 64-99. Appleton-CenturyCrofts, New York, NY.

Robinson ES, Eagle DM, Mar AC, Bari A, Banerjee G, Jiang X, Dalley JW, Robbins TW. 2008. Similar effects of the selective noradrenaline reuptake inhibitor atomoxetine on three distinct forms of impulsivity in the rat. Neuropsychopharmacology 33: 1028-1037.

Sara SJ. 2009. The locus coeruleus and noradrenergic modulation of cognition. Nat Rev Neurosci 10: 211-223.

Sara SJ, Vankov A, Hervé A. 1994. Locus coeruleus-evoked responses in behaving rats: A clue to the role of noradrenaline in memory. Brain Res Bull 35: 457-465.

Seu E, Lang A, Rivera RJ, Jentsch JD. 2009. Inhibition of the norepinephrine transporter improves behavioral flexibility in rats and monkeys. Psychopharmacology (Berl) 202: 505-519.

Southwick SM, Davis M, Horner B, Cahill L, Morgan CA III, Gold PE, Bremner JD, Charney DC. 2002. Relationship of enhanced norepinephrine activity during memory consolidation to enhanced long-term memory in humans. Am J Psychiatry 159: $1420-1422$

Sterpenich V, D'Argembeau A, Desseilles M, Balteau E, Albouy G, Vanderwalle G, Degueldre C, Luxen A, Collette F, Maquet P. 2006. The locus ceruleus is involved in the successful retrieval of emotional memories in humans. J Neurosci 26: 7416-7423.

Sullivan RM, Stackenwalt G, Nasr F, Lemon C, Wilson DA. 2000. Association of an odor with activation of olfactory bulb noradrenergic beta-receptors or locus coeruleus stimulation is sufficient to produce learned approach responses to odor in neonatal rats. Behav Neurosci 114: $957-962$.

Taylor JR, Olausson P, Quinn JJ, Torregrossa MM. 2009. Targeting extinction and reconsolidation mechanisms to combat the impact of drug cues on addiction. Neuropharmacology 56 (Suppl. 1): 186-195.

Usher M, Cohen JD, Servan-Schreiber D, Rajkowske J, Aston-Jones G. 1999. The role of locus coeruleus in the regulation of cognitive performance. Science 283: 549-554.

Yang B, Wang Y, Cynader MS. 1996. Synergistic interactions between noradrenaline and glutamate in cytosolic calcium influx in cultured visual cortical neurons. Brain Res 721: 181-190.

Received July 1, 2010; accepted in revised form November 10, 2010. 


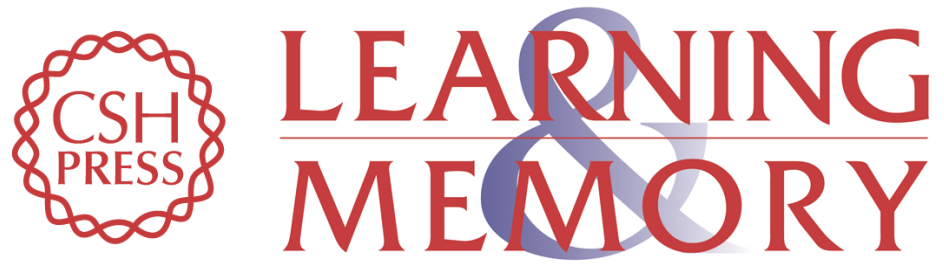

\section{Deepened extinction following compound stimulus presentation: Noradrenergic modulation}

Patricia H. Janak and Laura H. Corbit

Learn. Mem. 2011, 18:

Access the most recent version at doi:10.1101/lm.1923211

References This article cites 51 articles, 8 of which can be accessed free at: http://learnmem.cshlp.org/content/18/1/1.full.html\#ref-list-1

License

Email Alerting Receive free email alerts when new articles cite this article - sign up in the box at the Service top right corner of the article or click here. 